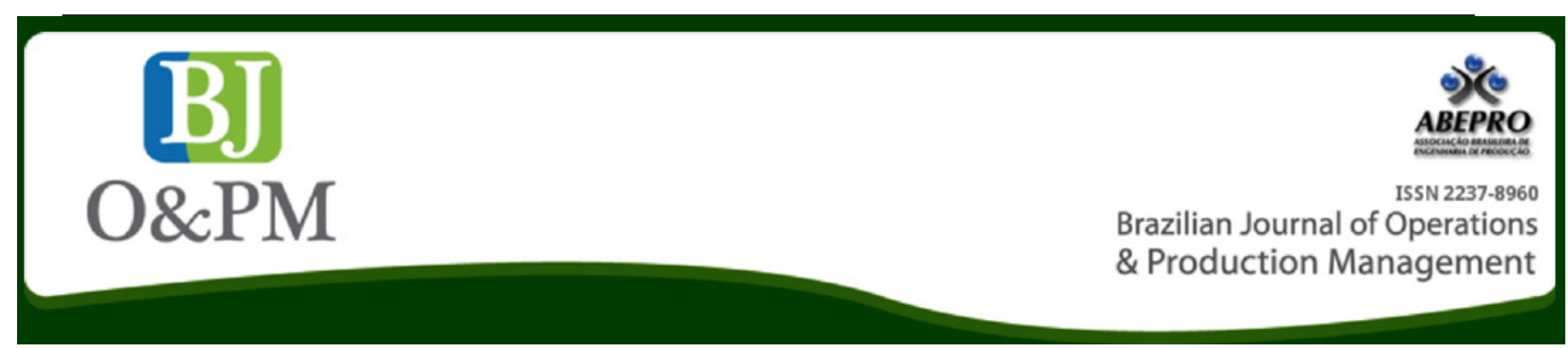

\title{
ROADMAP FOR THE IMPLEMENTATION OF A PROJECT MANAGEMENT MODEL IN A SME OF ENGINEERING AND TURN-KEY SUPPLY OF INDUSTRIAL EQUIPMENT
}

David Hermidaa; David de la Fuente ${ }^{a}$; Fernando García ${ }^{a}$

a University of Oviedo (UNIOVI) - Asturias, Spain

\begin{abstract}
Project management is focused on planning, executing, monitoring and controlling of all aspects of a project, defined as a temporary effort to carry out a unique result, in order to achieve the targets set under the criteria of time, quality and cost restrictions. In a small or medium-sized organization focused on this type of activity, the integration of the various factors involved in the project life cycle is needed. A roadmap developed as a set of guidelines for effective project management, tailored to this type of organizations but based on the existing sets of best practices and methodological standards (traditionally oriented to huge corporations), is pursued in this work through a comprehensive-qualitative analysis added to an interview approach.
\end{abstract}

Keywords: project management, roadmap, SME, small and medium-sized enterprises, management model 


\section{INTRODUCTION}

This work emerges from the idea of structuring the working method in a small or medium-sized organization dedicated to engineering and turnkey supply of industrial equipment and facilities, as a principle of operational improvement, by establishing a repetitive pattern based on a project management model, or "roadmap", capable of automating the creative work of the management process and the development of the productive work area, minimizing the human factors that may have some negative impact on both the project goals and the overall objectives of the company, and allowing better planning of the projects as well as the production area and the strategic objectives of the organization. In addition, the work also seeks further optimization of the roadmap by applying international standards for process integration.

Within an organization whose activity is focused on providing "turnkey" industrial equipment and facilities of a generalist character, which can cover many technical areas and adopt very different characteristics, often unique for each type of facility or equipment depending on the different conditions or on the needs of each client, the execution of the productive work must necessarily be oriented to projects.

This research starts with the assumption that the incorporation of management standards in project management can provide solutions to the problems of quality, time and cost due to the lack of integration of the management system, which can cause numerous problems in projects along its entire value chain. The control of information flows and relationships between the various participants (including their cooperation grade and the ability to forecast the impact of their decisions) directly affect the level of quality, time and cost of the project from the initial time.

The main objective of this work is to establish an optimized set of guidelines which integrates a subsequent flowchart (roadmap) as a model adapted to the initiation, planning, execution, monitoring and closing of projects in small and medium-sized companies, optimized by applying the best practices contained in the most-globally used methodological standard (PMBOK), and tailored to the particular organization type. Therefore the sub objectives will be:

- Automate the "creative part" of the management work process in the production area;

- Minimize possible human factors with negative impact on project objectives;

- Allow better planning and achievement of the objectives of the organization.
The PMBOK standard for Project Management (developed by the Project Management Institute - PMI, USA) is aimed at defining key variables and processes that make up the system described, and it is taken as reference basis for the realization of the model and subsequent optimization, in order to identify the processes, the relationships between them and the information flows.

This work is limited at first to a small or medium-sized company dedicated to the development of turnkey projects and technical consulting with a clear orientation towards qualitative growth in technology and product market. The company would develop its work in the field of engineering services and supply of equipment and industrial installations in multiple productive, environmental and civil applications.

In the particular case of this study, the target company would have a workforce of between 40 and 50 people in total, with an annual turnover of 5-6 million. Although different countries and regions mark different dimensional limits for the definition of small and medium-sized companies, most of them are aligned in the dimensional limits of our target company would find approximately the border between the definition of small business and medium-sized. However, the purpose of the work aims to be provided with sufficient scalability for subsequent extrapolation to larger firms.

The overall organization of the production area of the target company would present an almost horizontal organizational structure with a project-oriented functional structure. The structure of the Production Department of the company would correspond to a matrix structure closer to a project-oriented more than a classical functional structure, and would present two main areas: one focused on project management (hereinafter GP) and the other focused on the engineering and technical development (hereinafter DT) that would support the management area.

\section{METHODOLOGY}

Despite there are many existing models of processes, procedures, guidelines and tools covering various aspects of project management, they have often focused on large companies and corporations, making it necessary to adapt themselves to the particular conditions of small and medium companies. Furthermore, the peculiarities of the industry, and the characteristics with respect to corporate management of organizations dedicated to engineering and consultancy, further accentuate this adaptation needs.

In small companies (micro-sized), management approaches are usually more "familiar" with autocratic management styles, far from the norms and procedures of the existing approaches to project management. As management methodologies in organizations of increasing size (medium) are analyzed, with a more intensive use of 
specialist staff, the formal coordination requirements are also higher, pointing to a greater willingness to implement planning systems typical from project management approaches and greater involvement in the planning and management areas by the staff of the organization, which usually looks for more democratic management styles.

Within this need to coordinate the work of specialists and therefore to integrate formal processes of an increasing degree of sophistication, is that small and medium sized companies require a customized project management model which allows such formal coordination, but lightened in comparison with standard methodologies used in large companies and corporations, whose degree of complexity and bureaucracy could be an impediment on productivity and achieving goals.

This research tries to find a project management model materialized in the form of a roadmap (represented as a flow chart of processes) that fits an optimal level of sophistication and allows formal project management in an organization as the one described, picking only those aspects of increased use of the most common methodological standards.

After conducting a comprehensive review of the framework for managing projects, an analysis of the different approaches and its degrees of tailoring to SMEs (small and medium sized companies), and of the relationship of these approaches with the different cultures of the organization, is performed. An analysis of the relationship of that framework to process-based approaches (like EFQM), and to the innovation management methodologies, is also performed, as well as the relevance in that framework of existing tools and information technologies is also considered under the cross objective of obtaining patterns adapted to the objectives of the organization, enabling continuous improvement.

We have conducted therefore (as the main method for this work):

- A comprehensive review of the framework for managing projects

- An analysis of the different approaches and its degrees of tailoring to SMEs

- An analysis of the relationship of these approaches with the different cultures of the organization

- An analysis of the relationship of that framework to the process-based approaches and to the innovation management concepts

- A study of the relevance in that framework of existing tools and IT technologies (obtaining patterns adapted to the objectives of the organization)

\section{QUALITATIVE ANALYSIS AND REVIEW}

The Project Management Institute (PMI) is one of the largest professional associations in project management worldwide. PMI is the organization responsible for the standard PMBOK (Project Management Body of Knowledge), which includes a body of standard knowledge in project management as an agreed set of best practices. PMBOK is the standard most extensively used in the world, and therefore will be the standard under which the roadmap for project management in small and medium sized companies proposed in this paper will be developed.

Nevertheless there are multiple recognized bodies in the world that have articulated bodies of knowledge and standards with different characteristics, such as the IPMA, the APMG (PRINCE2) or the associated methodologies and systems Lean / Six- Sigma, Agile or Scrum.

The international standard ISO 21500 also provides guidance on the concepts and processes related to project management that are important for the projects and have an impact on the performance, being mainly addressed to managers and project teams.

In any case, despite the high degree of expansion of quality standards and quality management methodologies in almost all types of production and service companies, it is not the same in the field of project management and even the quality management within projects. Having some rules and standards that serve as methodological guides in those areas, they have not achieved a level of expansion as quality standards (especially ISO 9001), a situation that is even more accentuated in the case of Spanish small and medium sized companies.

There is a clear relationship between the successful execution of projects and the adaptation of the methodologies, or what is commonly referred in English as "tailoring", which refers to the degree to which a methodology is designed to suit the organization and projects developed.

As stated within a study titled "the art of tailoring" (Whitaker, 2012), after a review of the nature of the methodologies and their importance in organizations, a problem is located in the interest of organizations to obtain and implement methodologies "caught on a shelf" previously designed for direct application in the framework of projects, assuming the payment of the license and accreditation of people will be sufficient for the methodology to fit well on their organizations, but often finding that the methodology is not used or is used with a low degree of maturity.

The aforementioned study raises the alternative of defining a management methodology adapted itself or "tailored" to better measure the organization, using the 
Brazilian Journal of Operations \& Production Management

Volume 13, Número 3, 2016, pp. 252-262

DOI: 10.14488/BJOPM.2016.v13.n3.a3

same language of the organization. It identifies the need for people with adequate levels of expertise and commitment, and to iterate the design process to ensure that the methodology is correct.

For obtaining that degree of adequacy, three fundamental phases are marked: the first is to select the elements that will form the methodology from a body of knowledge (such as PMBOK), choosing the process tools and techniques that are appropriate for the style of project, size and complexity, serving as the basis for the project management methodology. The second is to make the adjustment by selecting which elements of the methodology apply to the particular project, which may involve the project manager and the project management office (if exists). The third is made during the proper execution of the project, iteratively checking what particular combination of elements remains appropriate, and adding the lessons learned. Some authors (Turner et al., 2010) propose -in their particular work- as the next step, and in addition to outline their results through quantitative surveys, to develop that lightened approach to project management adapted to small and medium sized companies.

Therefore it exists already in literature some works in which project management models for small and medium sized companies in the engineering services industry are developed. Usually they consider as the most important points the definition of internal procedures for information feedback from completed projects (lessons learned) and a management structure to support -and properly controlhow to assign different types of resources, sometimes based on the theoretical aspects and technical regulations like PMBOK or other formal methodologies such as PRINCE2 (both complementing each other).

The culture and style of the organization also affect the project ability to achieve the objectives. Many organizations have developed business cultures with visions, values, shared norms, policies, methods and procedures, work ethics etc. The culture of the organization is considered to be an "environmental factor" of the company, so that different cultures and styles of the organization should be known by project managers because they can influence them. There are also some hybrid models which established the links between management processes (like the EFQM approach) and project management (Monterrey, 2006).

These models establish how to allow evaluating, monitoring and improving company programs and improve the methodology for measuring results and consistency with objectives. Following that way, they could improve in the medium term the results of key indicators and reformulate the process map of the management model used, focusing the problem in the organizational structure of the company (as they could be focused on process management approaches, while the methodology for the regular work was done through projects, something that is also frequently found in SMEs).

All these issues need to establish methodologies, process approaches and management models adapted to the company in line with accepted standards which ensure the control of project management methodologies. The existing research in this field by the organizations is very low, being more associated to work groups specifically dedicated to this issue (as the Project Management Institute). However, the lack of organizational models in project management has a clear impact on the overall goals of SMEs, directly affecting the degree of success of the projects.

In the environment of small and medium sized organizations, there are also links between innovation management and the internal management systems, the organization, and the ways of implementing the operations and projects. Some studies have developed models of management of technological innovation based on systemic approaches to measure the impact of innovations in small and medium companies. A notable example is the case of Ortiz (Ortiz, 2007), whose general model is based on the balanced scorecard, designed according to the characteristics of SMEs (in the case of manufacturing SMEs in Latin America) from which it is determined that the key decisions on these companies is to invest for the generation of innovations. The model allows simulation using system dynamics software for validation.

Other studies such as "Dynamic analysis of a research and development project' (Alvarez, 1997) also used a systemdynamics simulation model for the design of models applied to the research and development project management, which contributed to a significant improved knowledge on the management of such projects by empirical studies. The integration of the different value chains involved in the life cycle of the project is necessary for a project management configured efficiently and effectively (Ballard and Howell, 1998). Therefore, the incorporation of new paradigms in project management provides solutions to the problems of quality and costs due to the lack of integration.

\section{RESULTS AND OPTIMIZATION}

Through this way, an initial approach to the operational definition of the roadmap is developed, establishing a flowstructured program for implementation and monitoring processes, analyzing their strengths and weaknesses. Currently a second round of feedback is being prepared throughout an interview to experts (this stage is detailed below). It is intended to establish the basic outline of the roadmap for project management in small and medium- 
sized companies of engineering and turnkey supply of industrial equipment and facilities, by defining each of the operational guidelines to be followed for the achievement of the project objectives.

Likewise, a series of underlying templates for support of management control according to defined structure are developed:

- Roadmap Control Template

- Planning Control Template

- $\quad$ Assets Control Template

- $\quad$ Checkpoints Template

- $\quad$ Technical Assessment Template

In addition to the characteristics of the defined target company, and after the formal review and qualitative analysis of the relationship between the management framework with the rest of the points (tailoring, cultures of the organization, process-based approaches, innovation, IT alignment), the following working hypothesis are added as a starting scheme that serves to consider the most common cases:

- The company does not have specific software for documentation management. The organization covers that issue with a common procedure based on each project (work order) would be associated to a computer folder (named with a code).

- However the company will have a management computer software (which could be based on a commercial ERP software or as a tool expressly designed or configured) to support the roadmap in several respects.

In addition to this roadmap is also proposed a flexible control scheme of meetings, in order to supplement its capacity in the areas of monitoring, improving the participation of the management board, and enhance the contribution with strategic and operation ideas of the Production Department, as well as the collaboration of the different members of the project team in optimizing the management and the definition of internal improvement processes.

Below the visual map shows the basic outline of the roadmap for project management, so that each of the operational guidelines (previously defined) remain represented in a flow-structured program that allows adequate traceability during execution of the project. Each of the items / guidelines of the roadmap scheme is biunivocally identified (in its logical order of execution) by a sequence represented by the letters of the alphabet, from $A$ to $Z$, as it is shown in the Figure 1, Figure 2, Figure 3 and Figure 4:
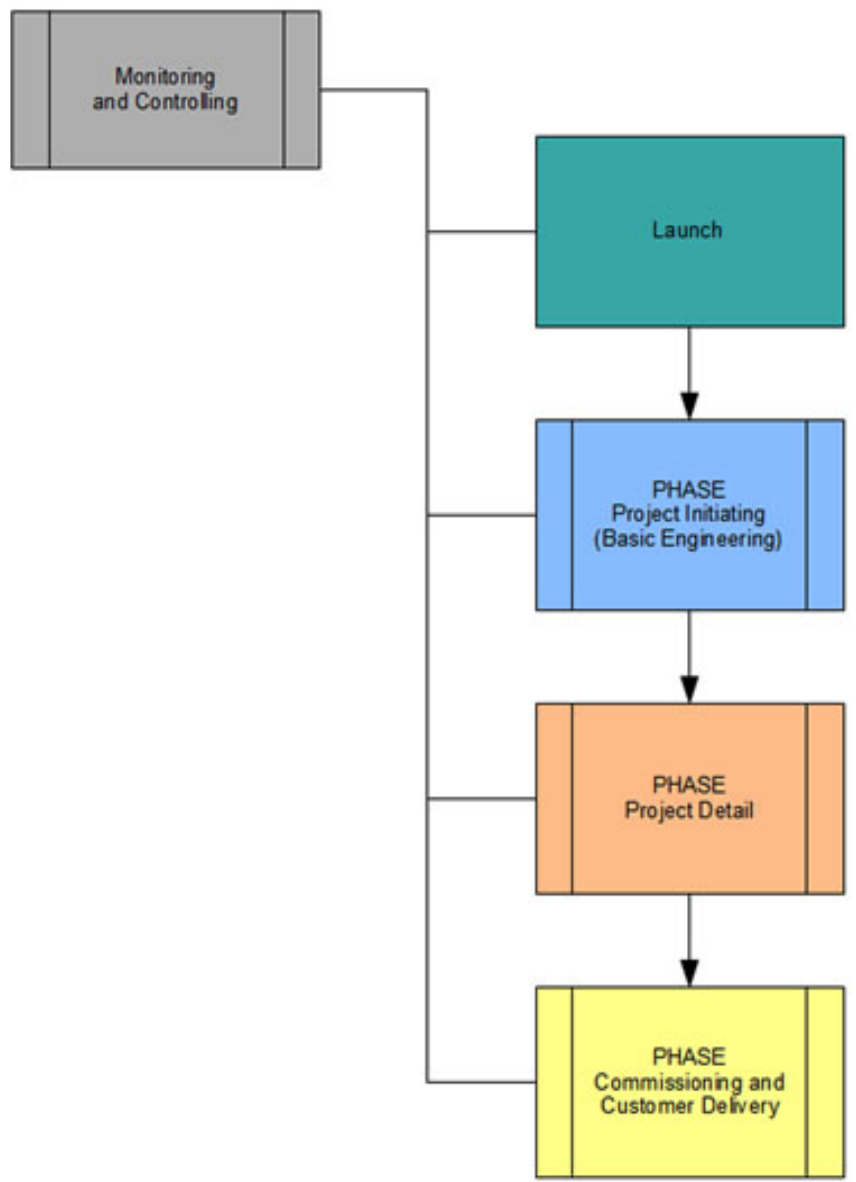

Figure 1. Roadmap Main Scheme Source: The authors own.

The organization fits within the area of GP all the work of project initiation and development of basic engineering as well as the approval of the detailed engineering and technical specifications (developed in this case within the area of DT ), the executive decision on contracting, order tracking and manufacturing workshops control, assembly and commissioning monitoring, financial control and monitoring of external and internal billing and cash flow, and customer relationship, since the project managers are the highest responsible for the project, and all decisions to meet customer needs and expectations are based on their figure when it comes to time and quality within the technical and budgetary restraint that marked the contractual agreements.

This study is focused on the integrative part of the PMBOK standard as a basis for optimizing the route map developed, introducing all key points that were collected in a previous analysis of the standard within the knowledgearea of integration management throughout all groups of processes, from initiation to project closure. Then the study enters the integration process of developing the management plan of the project, which could be considered the mainstay in the management model, since it "wraps" all 


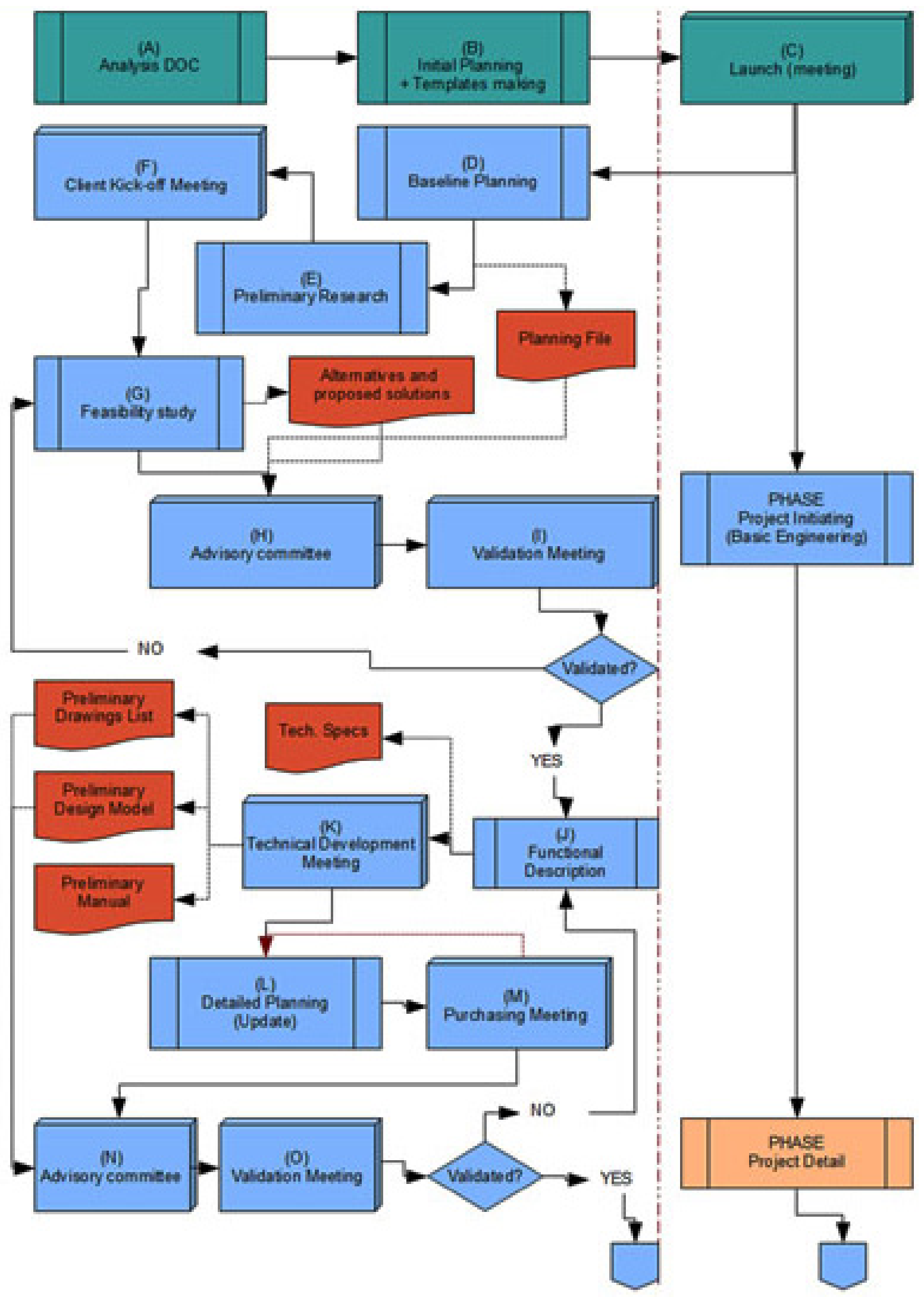

Figure 2. Roadmap Visual Map 1/3 Source: The authors own. 

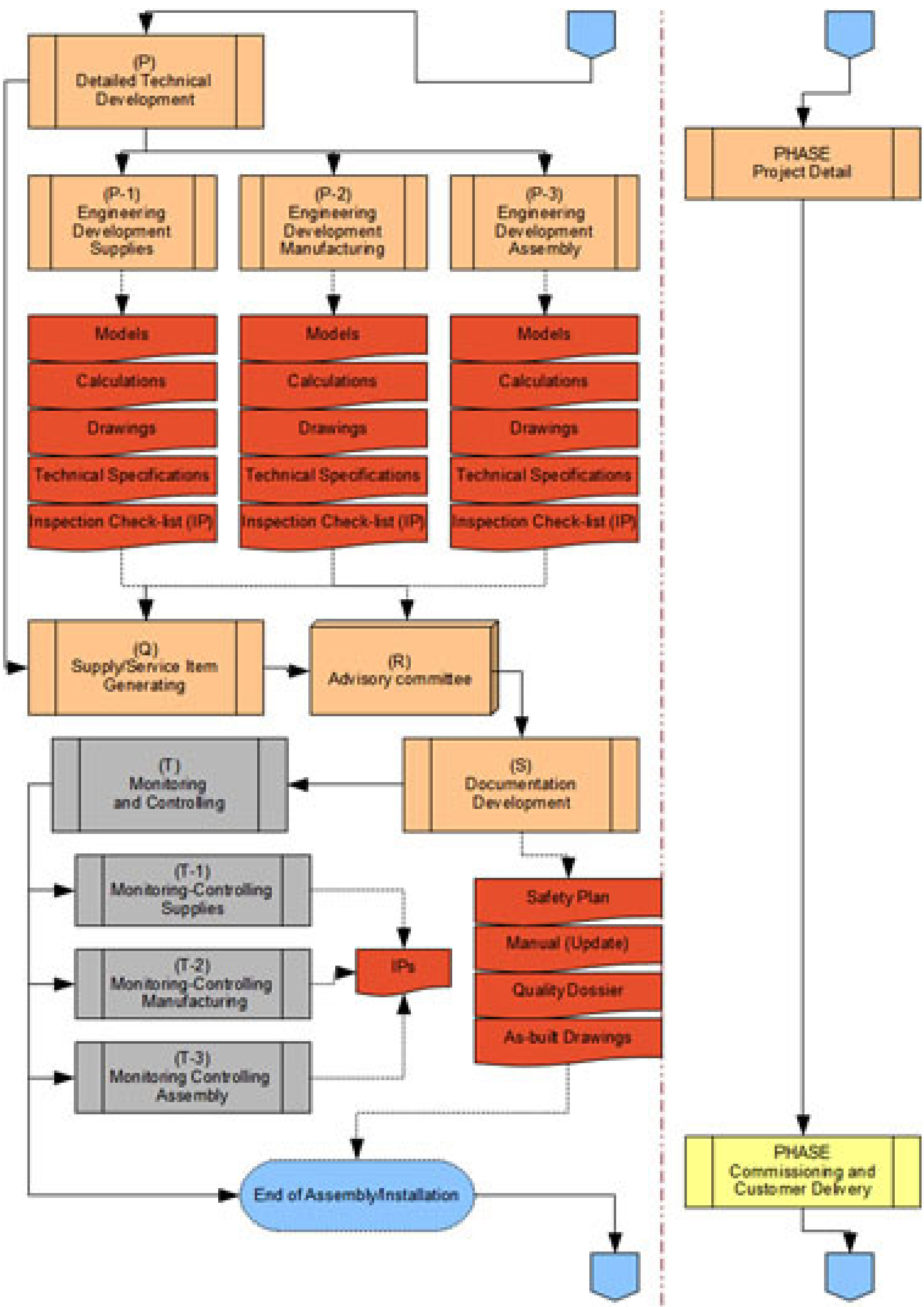

Figure 3. Roadmap Visual Map 2/3

Source: The authors own. 


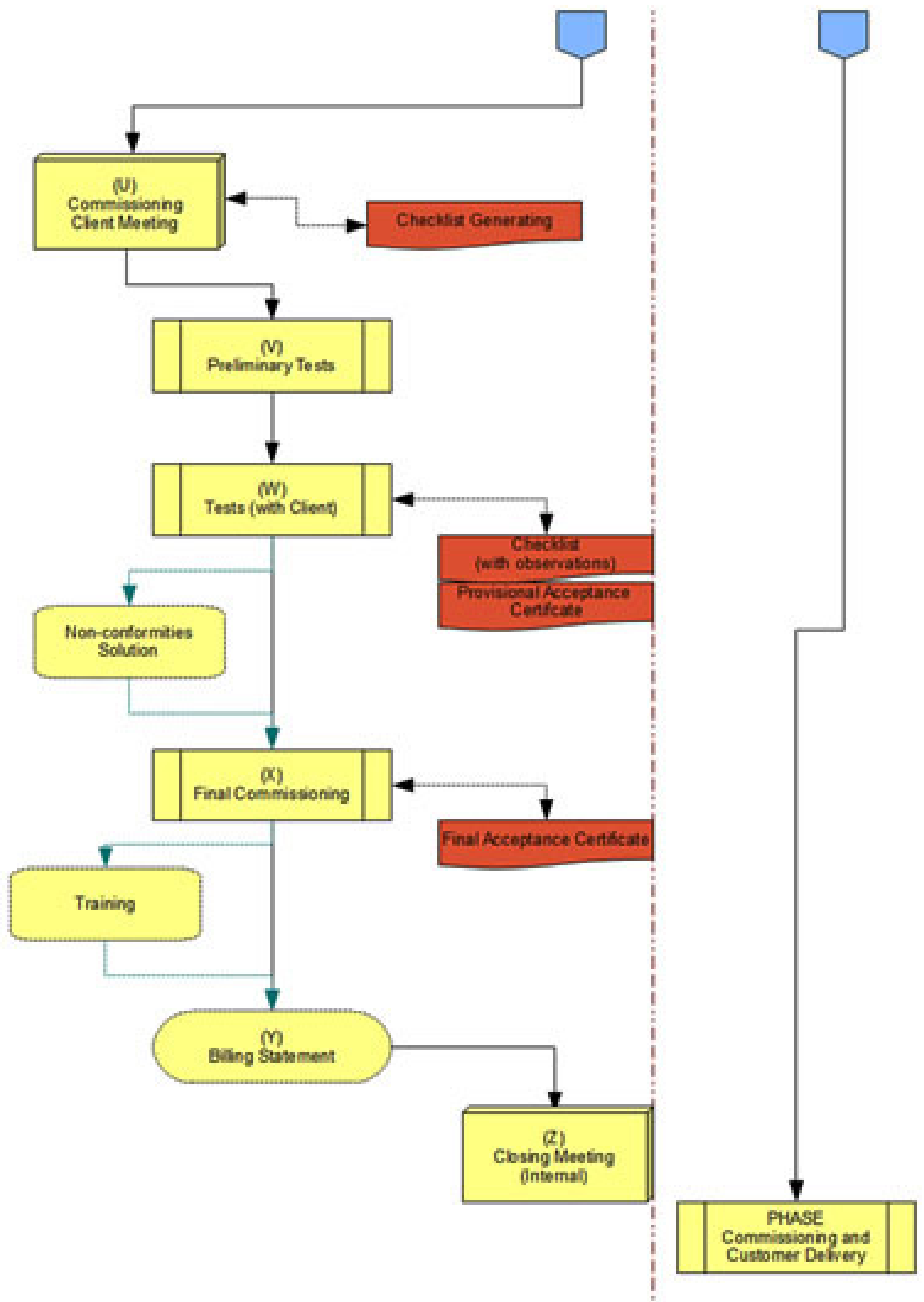

Figure 4. Roadmap Visual Map 3/3

Source: The authors own. 
processes related to planning across every knowledgearea presented in PMBOK: scope management (scope planning, detailed requirements collection and definition, scope definition through a work breakdown structure WBS), time management (time planning, definition and sequencing of activities, resource and duration estimations, and schedule development), cost management (cost planning, cost estimates and budget development), quality planning and management, human resources management, communications management, procurement management, stakeholder management, and risk management (planning, identification, qualitative and/or quantitative analysis and definition of response plans).

The roadmap route is designed to bring together and document, as marked on the standard, the necessary actions for the integration and coordination of the planning on all essential knowledge-areas, such as the scope (through the study of design alternatives within a "tree" that responds to each of the "blocks" of the work required and carry out a complete functional description of the product or service), schedule and budget, and it is performed on all key processes including its gradual nature (reappearing in the relevant points that may require some upgrade). While most of the processes associated to the subsidiary plans are implicitly considered in each of their counterparts in the roadmap, a need to emphasize the relative sequencing and resource/durations estimation within the schedule section and cost estimation was found.

A Responsibility Assignment Matrix (RAM) is also used to show the relationships between activities or work packages and project team members. The matrix format shows all activities associated with one person and all people associated with one activity. This also ensures that there is one person in charge to account for a given task in order to avoid confusion. An example of RAM is a RACl diagram, which means "Responsible (R), Accountable (A) Consulted (C), Informed (I)" and that is one of the key elements in the multiple component of role assignment within the Early Equipment Management, or EEM methodology, which is related to the World Class Manufacturing - WCM (a popular Japanese model of overall excellence in the logisticsproduction cycle, born in the Toyota Corporation, and linked to concepts and strategies such as Total Productive Maintenance - TPM, Total Quality Control - TQC, and Just in Time - JIT methodologies, as well as some aforementioned tools like Lean / 6 Sigma, 5S, FMEA, Kanban, Kaizen etc. and aims to foster an optimal level of competitiveness in industrial production) which generates a process (workflow) that tries to minimize the time to start new investment projects and also to optimize their recurring costs.

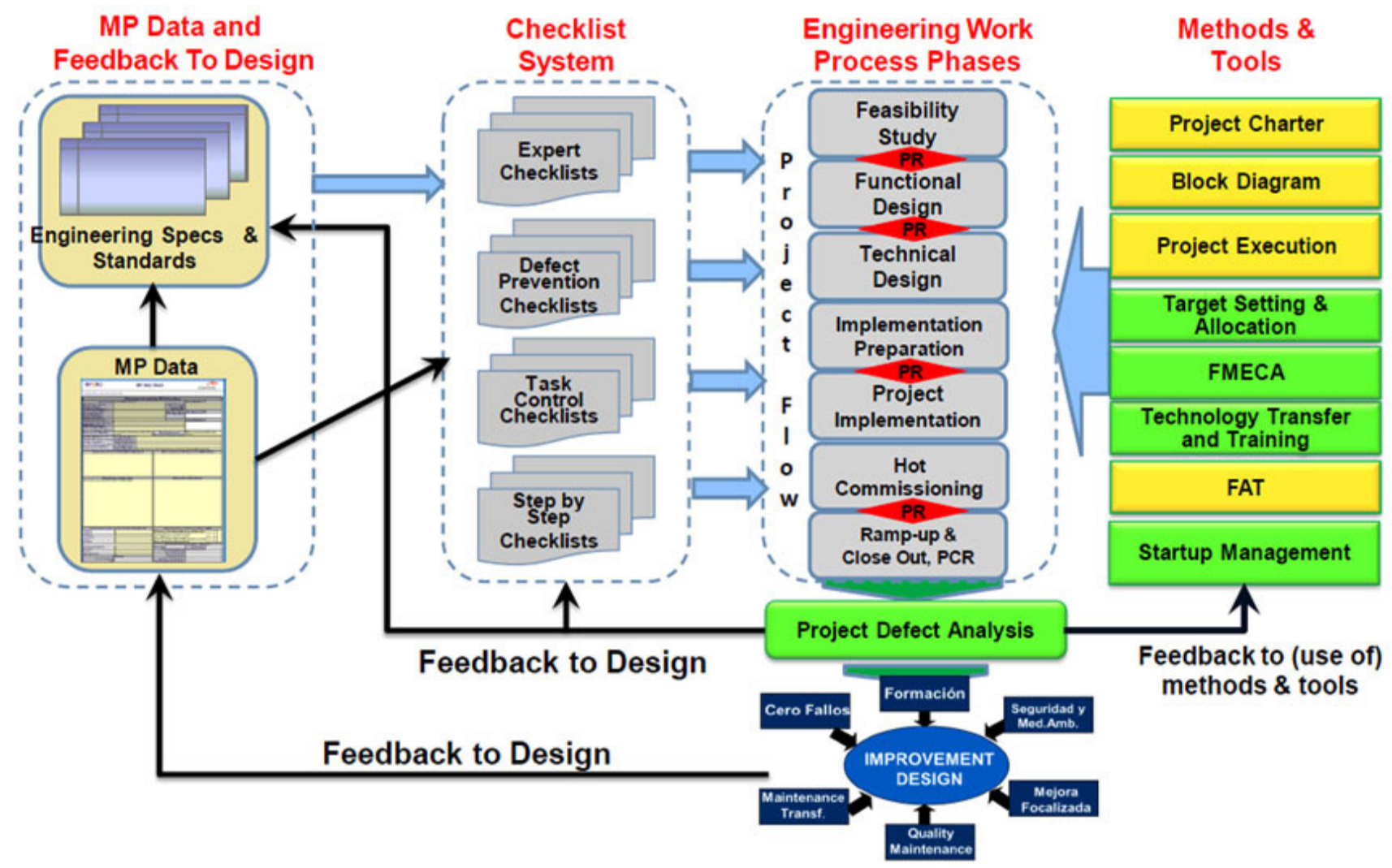

Figure 5. EEM System Scheme Source: The authors own. 
The workflow of EEM has direct application in engineering projects and is used by many corporations, finding a direct synergy with the project management roadmap as can be seen in the Figure 5 shown below, which shows a global and schematic diagram of the EEM system (diagrams blocks, feedbacks between processes to optimize designs, specifications and standards, checklists generation, project constitution, implementation etc.).

In order to close the chapter of optimization based on the use of the PMBOK standard, it is important to consider that, in the field of project management, there are many activities that have very similar features, as every case there is an objective and a forecast of what will be needed (cost) to get a later stage in which a certain amount of resources (execution of work), and a result in terms of achieving this objective. Given that there are many projects that fail (in the sense of exceeding the term or the expected cost, or get a lower delivered product quality and customer satisfaction) and is important to understand the aspects related to the achievement of a project, aspects which seem to be largely related to the intrinsic ability of the project team in terms of supervision, monitoring and control of the work performed, which at the same time is related to the dynamics of the errors in performing of the various tasks, and changes control associated with such errors.

This hypothesis is considered in this work by using a model that, using system dynamics, helps in how to understand these aspects (a tool widely used in the field, as described in the review of the state of the art, for its proven usefulness in solving problems related to an inaccurate or poorly quantified reality, but complex and subject to the intervention of human decisions, such as the ability to manage projects).

The model proposed (Figure 6) conceptualizes the simplest structure of the problem and then is improved gradually. For this it relies on the use of a software tool (PLE Vensim v6.3D, Ventana Systems, Inc.) capable of simulating and displaying the results of the changes in the model structure, allowing thereby to verify the approach described.

In this case it is possible to appreciate the phenomenon of accumulation of errors in the final stages of the project
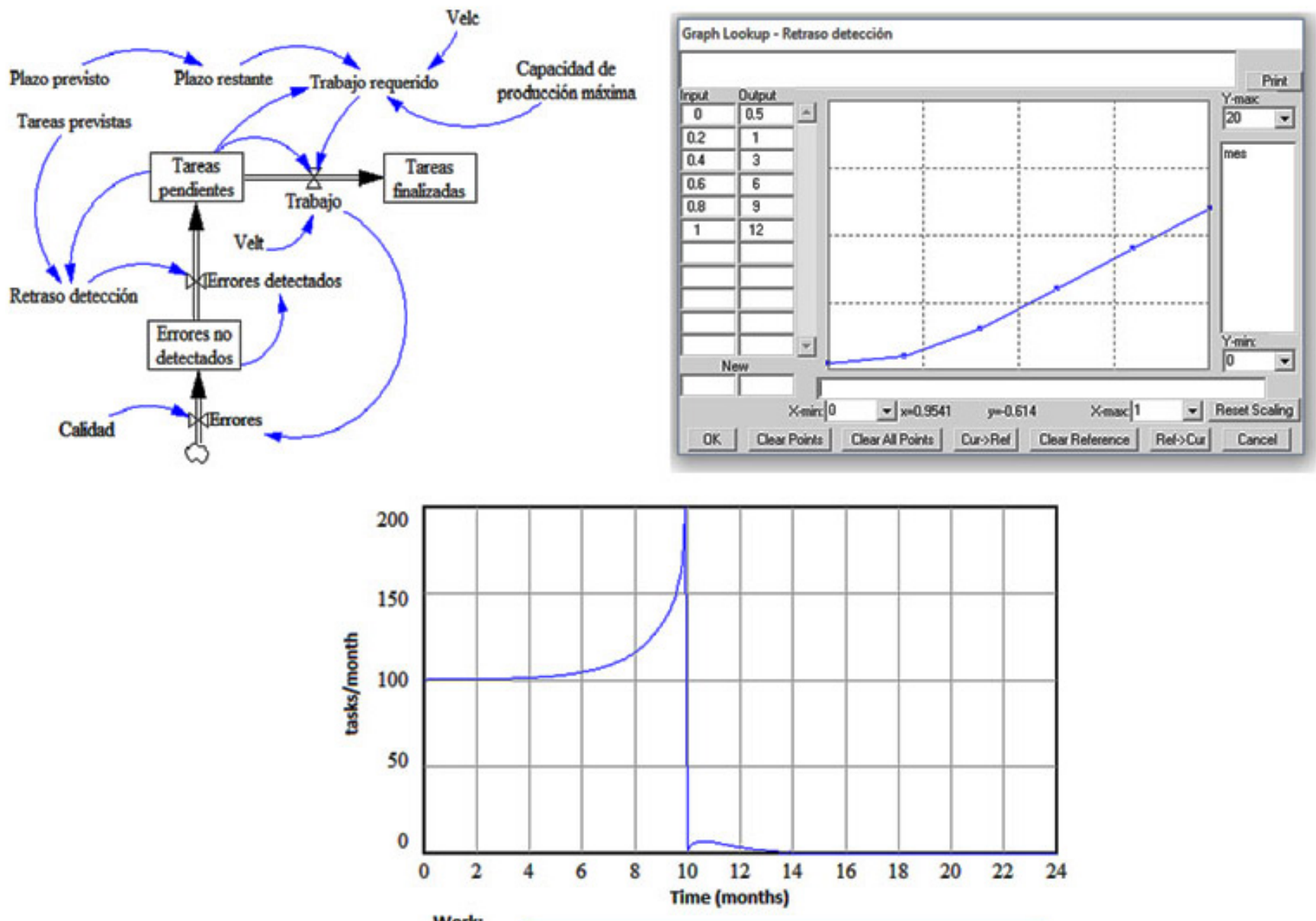

Figure 6. System Dynamics Model \& Simulation Source: The authors own. 
and how the distribution of tasks is progressively increased (despite the existence of a planning and scheduling of workloads) extending beyond the date of delivery of the project.

This type of dynamic simulation models also allows analysis of sensitivity in response to different changes in their parameters, for example, it is possible to check -in the model described- how a change in the expected duration does not change the dynamic behavior of work load, but only approaches or moves away the phenomena of accumulation of tasks.

With the aim of going a step further in the optimization of the roadmap defined within the one provided by the application, adapted in scale, of a standard in project management (PMBOK), we have tried to go in depth into the field of good practices by consulting the opinion of experts in the field and staff with experience in project management in these type of organizations.

Compression of the problem which these organizations have to face is the first step necessary to develop a management model, which has been the main objective of this work, and to define a survey for the second optimization stage. It is therefore common to use a multitude of mechanisms such as focus groups and surveys in which professionals, members, volunteers and others are involved, such as the Project Management Institute itself has carried out through the Standards Development Committee to develop, for example, the ethical code of conduct.

The survey is structured along each of the 27 points of the roadmap, contemplating a key question for each of the steps of the route structure. The largest proportion of the responses will carry the decision on whether to require modification, in contrast also with the detailed analysis of the changes arising from the PMBOK application.

\section{CONCLUSION}

The PMBOK standard is the result of many years of progress and improvement of a body of knowledge with a high degree of detail between a massive number of professionals in the field of project management, which provides with an undeniable degree of rigor and precision. However, its own magnitude has caused a very high level of sophistication, and thus the level of complexity requires a great deal of effort, time and other resources for its effective implementation.

There is no doubt that the establishment of procedures and standards of management is an essential requirement for the effective and successful project management. However, in a small or medium sized company of engineering and technical consulting, on one hand (and as was detected in the state of the art review) the organizational structure pushes management methodologies to a process approach, even if the work is carried out in the form of turn-key projects. On the other hand, the natural shortage of resources makes difficult (often impossible) the necessary investment of resources and time needed for implementation.

In this context and due to the implicit nature of this type of organization, the need for a process approach arises adapted in its own sophistication grade to the typical available resources, allowing the feasibility of the process implementation.

Although there are existing process models and guidelines covering various aspects of project management, they are usually focused on large companies, making necessary to adapt them to the particular conditions of SMEs. In addition, the particularities of the industrial sector and of the organizations dedicated to engineering (regarding management), further accentuate this adaptation needs, which can be achieved through easy-to-install roadmaps for management as the one proposed.

\section{REFERENCES}

Álvarez, V. (1997), Sistema de gestión de flujos de y reingeniería de procesos. Universidad de Oviedo, Spain

Ballard, G. and Howell G. (1998), What Kind of Production Is Construction. Sixth Annual Conference of the International Group for Lean Construction Proceedings, Brazil

Monterrey, M. F. (2006), Establecimiento de un modelo de gestión híbrido entre la gestión por procesos y la gestión por proyectos y generación de herramientas de apoyo en la Agencia Local de Promoción Económica y Empleo de Gijón. Universidad de Oviedo, Spain

Ortiz, F. (2007), Modelo de Gestión de Innovación Tecnológica en PYMES. International Conference on Industrial Engineering \& Industrial Management

Project Management Institute (2004), A guide to the project management body of knowledge. Newtown Square, $\mathrm{Pa}$, Project Management Institute.

Turner, R., Ledwith A., and Kelly J. (2010), Project management in small to medium-sized enterprises: Matching processes to the nature of the firm. International Journal of Project Management, Vol. 28, No. 8

Whitaker, S. (2012), The Art of Tailoring: Making your Project Methodology Fit. Project Management Institute Global Congress Proceedings, Vancouver, Canada 